\title{
Hightlights from this issue
}

doi:10.1136/gutjnl-2013-304904

\section{Luminal GI \\ Benefits of mass eradication of $H$ pylori infection}

What are the benefits and risks of mass eradication of $H$ pylori infection in the community? In this issue of Gut, Lee et al attempt to answer this question with a unique study from Taiwan. Mass eradication (chemoprevention) of $H$ pylori infection was started from 2004 for 5000 residents of Matsu Island who were $>30$ years of age. The main outcome measures were changes in the prevalence and incidence of $H$ pylori infection and premalignant gastric lesions before (1995-2003) and after (2004-2008) chemoprevention. Eradication of $H$ pylori infection substantially reduced the incidence of $H$ pylori infection, gastric atrophy and peptic ulcer disease within the relatively short time
Emad El-Omar, William Grady, Alexander Gerbes, Editor and Deputy Editors period of this study. The intervention did not reduce the incidence of intestinal metaplasia or decrease its histological severity, supporting the "point-of-noreturn' theory. There was an increase in incidence of oesophagitis after intervention (see table 1). This finding, in a population where gastric atrophy was prevalent, suggests a causal relationship between eradication and onset of oesophagitis. The findings of this study support the use of this strategy to prevent gastric cancer in populations where $H$ pylori is endemic and the incidence of gastric cancer is high (see page 676).

\section{Is choice a good thing?}

It is well known that screening prevents colorectal cancer, yet many people elect
Table 1 Changes in the prevalence of gastric and oesophageal lesions before and after mass eradication of Helicobacter pylori infection

\begin{tabular}{lcc}
\hline Outcome variable & Before chemoprevention & After chemoprevention \\
\hline Number of population & 1762 & 841 \\
Gastric atrophy & $1056(59.9)$ & $115(13.7)$ \\
Intestinal metaplasia & $558(31.7)$ & $327(38.9)$ \\
Indefinite for dysplasia & $142(8.1)$ & $128(15.2)$ \\
Low-grade dysplasia & $6(0.3)$ & $17(2.0)$ \\
High-grade dysplasia & $0(0)$ & $0(0)$ \\
Active peptic ulcer & $193(11.0)$ & $30(3.6)$ \\
Reflux oesophagitis & $241(13.7)$ & $230(27.3)$ \\
Los Angeles grade A & $177(10.0)$ & $181(21.5)$ \\
Los Angeles grade B & $58(3.3)$ & $38(4.5)$ \\
Los Angeles grade C & $6(0.3)$ & $10(1.2)$ \\
Los Angeles grade D & $0(0)$ & $1(0.1)$ \\
Barrett's oesophagus & $0(0)$ & $2(0.2)$ \\
\hline
\end{tabular}

Values are number (\%).
H

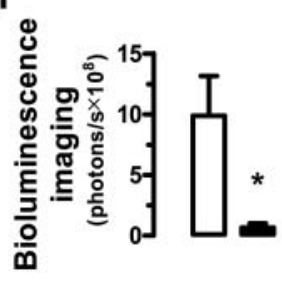

LV-miR-shRNA-ctrl
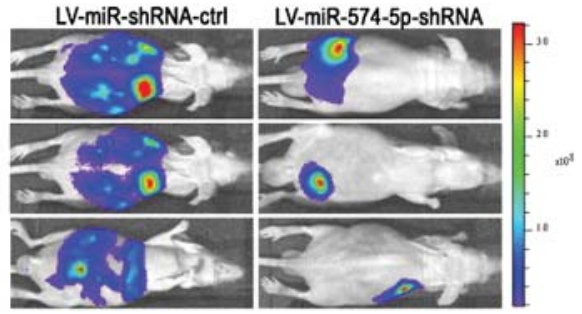

Figure 1 (H) Intraperitoneal tumour growth as demonstrated by bioluminescence imaging in the live nude mice inoculated with CT26 cells stably-overexpressing a luciferase reporter gene 24 days after treatment with shRNA lentivirus that suppressed miR-574-5p. This figure is only reproduced in colour in the online version. not to participate in screening programmes. The noncompliance with screening recommendations is related to a variety of factors including fear of endoscopic examinations, convenience, and the aesthetics of stool occult blood tests. Surveys have suggested that screening rates would be higher if people were given a choice in the type of screening test they could have, but this has not been proven. Senore et al now show that screening rates do increase if people are given the choice of fecal occult blood tests if they decline flexible sigmoidoscopy. They found that participation rates increased from $39 \%$ to $55 \%$ and that this approach was effective in detecting more advanced adenomas and cancers. So, a strategy involving the sequential offering of flexible sigmoidoscopy and then fecal occult blood testing is feasible and improves colorectal cancer screening efficacy (see page 735).

Another advance in understanding the role of microRNA's in cancer

Colorectal cancers are known to have thousands of abnormally expressed genes, however, the cause of the gene deregulation and the nature of the effects of specific deregulated genes on the cancer's behaviour is often not known. One potential mechanism that may underlie gene deregulation in cancer is the aberrant expression of microRNAs. Yang and colleagues have conducted an elegant series of studies to assess the mechanism through which microRNA 574-5p promotes the formation of colorectal cancer. They studied tumours arising in mouse models of colorectal cancer as well as primary human tumours to find that miR-574-5p has increased expression in colorectal cancer and that this suppresses the expression of the RNA binding protein Quaking6/7 (Qki 6/7). Increased miR-574-5p also altered the expression of B-catenin and p27. These effects increased proliferation as well as migration and invasion and decreased differentiation. Together, their novel findings suggest that miR-574-5p is a potent ribo-regulator for $Q k i$ s and that aberrant miR-574-5p up-regulation can be oncogenic (see page 716). 


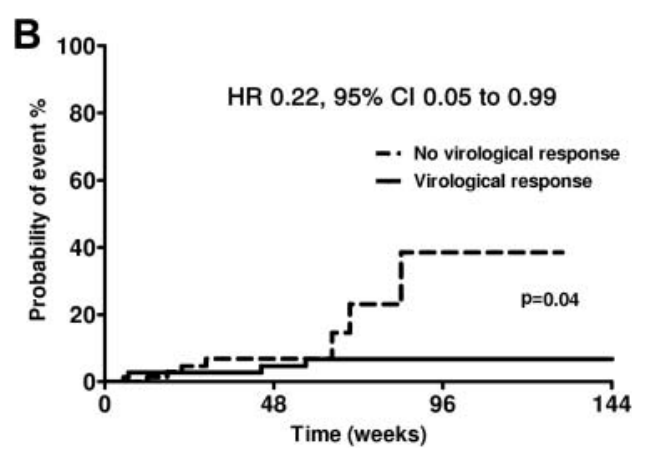

Figure 2 The Cumulative probability of developing a clinical complication during entecavir theraphy in patients with cirrhosis depends on virological response.

\section{Hepatology: Practical news for the treatment of HBV and HCV}

Virological response to entecavir improves clinical outcome in patients with HBV and cirrhosis

Chronic viral hepatitis B (HBV) is a major burden worldwide with an estimated half a million deaths annually due to complications of cirrhosis and hepatocellular carcinoma. Since these complications are correlated with $\mathrm{HBV}$ DNA levels marked reduction of HBV has been the prime goal. Entecavir (ETV) has proven very effective in $\mathrm{HBV}$ reduction and histological improvement of fibrosis. This interesting European multicenter cohort study (see page 760) shows that ETV also reduces clinical complications such as decompensation, hepatocellular carcinoma and death in patients with $\mathrm{HBV}$ cirrhosis (figure 2). Interestingly the probability of viral response was not impaired in patients with cirrhosis. Thus, complete HBV DNA suppression should be aimed for especially in patients with cirrhosis.

A novel inexpensive way to increase sustained viral response (SVR) in patients with HCV

Therapy of patients with viral hepatitis C (HCV) genotype 1 is still a major challenge due to poor sustained viral response (SVR) rates of the conventional treatment with pegylated interferon plus ribavirin and due to high costs and cumbersome practical issues of triple therapy. This interesting study from Italy (see page 766) suggests that vitamin B12 exerts anti $\mathrm{HCV}$ effects in vitro and in HCV genotype 1 patients. Monthly i.m. injection of $5 \mathrm{mg}$ vitamin B12 markedly increased SVR in these patients (figure 3). These exciting findings should now be confirmed by a randomised placebo controlled trial.

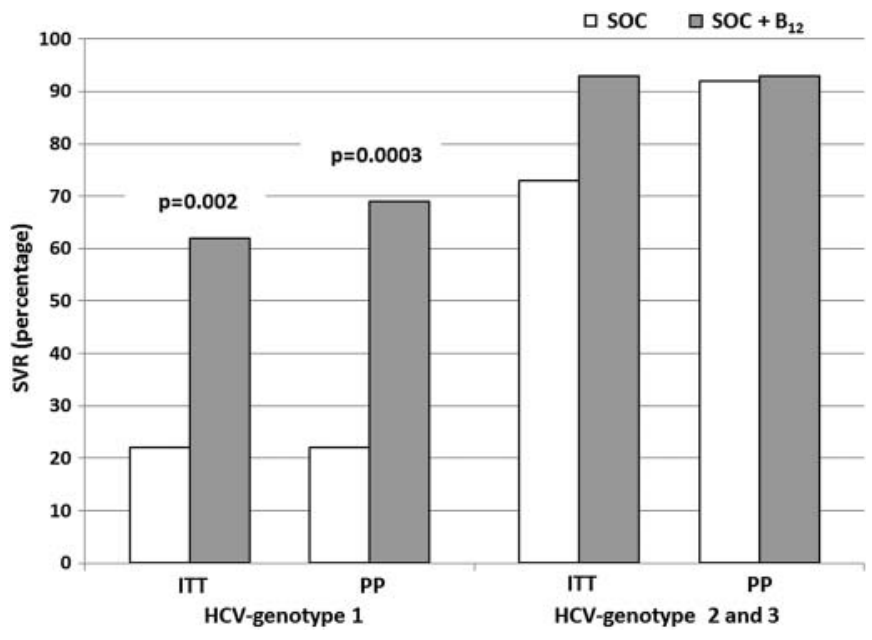

Figure 3 Sustained viral response (SVR) rates in patients who received standard of care (SOC) or SOC plus vitamin $B_{12}$ supplementation subdivided according to HCV genotypes. Fisher's exact test. ITT, intention to treat; PP, per protocol. 